\title{
Potential efficacy of weekly low-dose administration of bevacizumab as a combination therapy for platinum-resistant ovarian carcinoma: a retrospective analysis
}

Jin Suminokura' ${ }^{1}$ Morikazu Miyamoto ${ }^{1 *} \mathbb{D}$, Tomoyuki Yoshikawa², Hiroko Kouta ${ }^{3}$, Yoshihiro Kikuchi ${ }^{3}$, Taira Hada ${ }^{1}$, Hiroki Ishibashi ${ }^{1}$, Tsubasa Ito' ${ }^{1}$ Hideki Iwahashi', Soichiro Kakimoto ${ }^{1}$, Rie Suzuki ${ }^{1}$, Hiroko Matsuura', Naohisa Kishimoto ${ }^{1}$ and Masashi Takano ${ }^{1}$

\begin{abstract}
Background: Bevacizumab (Bev) plays the central role of the adjuvant therapy for patients with ovarian carcinoma. The aim of our study was to examine whether differences in the administration of Bev influence the prognosis of patients.

Methods: Patients with ovarian carcinoma who received treatment at two hospitals between 1999 and 2020 were identified. Patients treated with weekly low-dose administration of Bev (100 mg Bev on days 1 and 8 and $200 \mathrm{mg}$ Bev on day 15, monthly) at one hospital (group A) and those with monthly high-dose administration of Bev (15 mg/kg of Bev on day 1, monthly) at another hospital (group B) were retrospectively compared.

Results: Among the total patients, 44 were assigned to group A and 33 were assigned to group B. More patients in group A had advanced disease $(p=0.03)$ and a lower dose of Bev at the first time during the first cycle administration $(p<0.01)$ than in group B. Progression-free survival (PFS) was better in group A than in group B $(p<0.01)$. Multivariate analysis revealed that group A was a better prognostic factor for PFS (hazard ratio $0.53, p=0.03$ ). Stable duration was longer in group $A$ than in group $B(p<0.01)$. The incidences of adverse effects, including hematological toxicities such as neutropenia $(p=0.01)$ and nonhematological toxicities such as hypertension $(p<0.01)$, intestinal obstruction $(p<0.01)$, and thromboembolic events $(p<0.01)$, were lower in group A than in group B.
\end{abstract}

Conclusions: Weekly low-dose administration of Bev might improve prognosis and decrease the frequency of adverse effects associated with this drug although the prospective study was needed to get corroboration.

Keywords: Ovarian carcinoma, Bevacizumab, Administration, Prognosis, Adverse effect

*Correspondence: morikazu1118@hotmail.co.jp

${ }^{1}$ Department of Obstetrics and Gynecology, National Defense Medical

College Hospital, 3-2, Namiki, Tokorozawa, Saitama 359-8513, Japan

Full list of author information is available at the end of the article

\begin{abstract}
Background
Ovarian carcinoma is the most well-known gynecological cancer worldwide [1]. The standard treatment is the combination of debulking surgery and chemotherapy. Despite advances in treatment, approximately $75 \%$ of women with advanced disease experience recurrence, with worse prognosis [2].
\end{abstract} original author(s) and the source, provide a link to the Creative Commons licence, and indicate if changes were made. The images or other third party material in this article are included in the article's Creative Commons licence, unless indicated otherwise in a credit line to the material. If material is not included in the article's Creative Commons licence and your intended use is not permitted by statutory regulation or exceeds the permitted use, you will need to obtain permission directly from the copyright holder. To view a copy of this licence, visit http://creativecommons.org/licenses/by/4.0/. The Creative Commons Public Domain Dedication waiver (http://creativeco mmons.org/publicdomain/zero/1.0/) applies to the data made available in this article, unless otherwise stated in a credit line to the data. 
Bevacizumab (Bev) is a humanized vascular endothelial factor (VEGF) monoclonal antibody that inhibits tumor angiogenesis [3]. The Gynecologic Oncology Group (GOG)-218 and International Collaboration on Ovarian Neoplasms (ICON)-7 trials demonstrated that Bev as the first-line therapy improved progression-free survival (PFS) in patients with ovarian carcinoma $[4,5]$. In addition, the The Ovarian Cancer Study Comparing Efficacy and Safety of Chemotherapy and Anti-angiogenic Therapy in Platinum-Sensitive Recurrent Disease (OCEANS) and Avastin Use in Platinum-Resistant Epithelial Ovarian Cancer (AURELIA) trials showed that Bev improved PFS in patients with platinum-sensitive and platinum-resistant recurrent ovarian carcinoma [6, 7]. However, the main analysis of these studies showed that Bev could not improve overall survival (OS), although the subanalysis demonstrated that only the combination of Bev and paclitaxel could improve OS [8]. Recently, the Randomized, Double-Blind Controll Trial of Olaparib vs. Placebo in Patients with Advanced Ovarian Cancer (PAOLA)-1 trial indicated that Bev added to olaparib provided a considerable PFS benefit for patients with a deleterious germline breast cancer susceptibility gene (BRCA) 1 or BRCA2 mutation [9]. Therefore, Bev, an anti-angiogenic drug, plays an important role in the current and future treatment of ovarian carcinoma.

Anti-angiogenic drugs and chemotherapy drugs with anti-angiogenic effects have been reported to influence vessel remodeling according to different doses and durations of administration [10-12]. Until now, several reports have examined whether paclitaxel with anti-angiogenesis had antitumor effect depending on the differences of the administration [13-15]. However, no studies have examined the association between the schedule of Bev administration and the efficacy of the drug in patients with ovarian carcinoma. Therefore, the aim of this study was to investigate whether the schedule of Bev administration is related to its efficacy by comparing a monthly high-dose schedule and a weekly low-dose schedule.

\section{Methods}

This study was the retrospective analysis. Patients with ovarian carcinoma who received chemotherapy at Ohki Memorial Kikuchi Cancer Clinic for Women, and those who underwent surgery and received chemotherapy at the National Defense Medical College Hospital between 1999 and 2020 were identified. Among them, patients with platinum-resistant recurrence who received a Bevcontaining regimen were included in our study. Patients with a history of prior treatment with Bev, those with other carcinomas, and those receiving monotherapy with Bev were excluded.
In this study, the administration method of Bev were 2 patterns. At Ohki Memorial Kikuchi Cancer Clinic for Women, all patients received $100 \mathrm{mg}$ Bev on days 1 and 8 and $200 \mathrm{mg}$ Bev on day 15 monthly. No patients at this hospital received other than this method. They were defined as Group A. On the other hand, at the National Defense Medical College Hospital, all patients received $15 \mathrm{mg} / \mathrm{kg}$ Bev administered on day 1 monthly according to our previous report [16]. Patients at this hospital did not undergo other than this method. They were defined as Group B. The administration methods of Bev completely depended on the hospitals. The drugs co-administered with Bev at the two hospitals are listed in Table 1. The standard of the administration was set to the condition that met granulocyte count below grade 1, platelet count below grade 2 , hemoglobin level $>7 \mathrm{~g} / \mathrm{dL}$, and nonhematological toxicity below grade 1 . If patients did not meet the standard on day 1 of the next cycle or on days 7 and 14, the administration on day 1 was postponed by one week and the administrations on days 7 and 14 was skipped. Patients underwent these treatments for a maximum of six cycles unless unacceptable toxicities developed or diseases progressed. The clinical information of the patients was obtained from medical records. Staging was reevaluated using the 2014 International Federation of Gynecology and Obstetrics (FIGO) criteria [17]. Information on residual tumors was obtained from the operation records of the primary surgery. Response Evaluation Criteria in Solid Tumors version 1.1 was used to evaluate the efficacy of chemotherapy [18]. The state of the tumor was evaluated every $8-12$ weeks or when symptoms which was suspicious of diseases progression developed. Best response was defined as the most excellent response during treatment. The response rate and the clinical benefit rate was defined as the ratio of complete remission $(\mathrm{CR})$ and partial remission $(\mathrm{PR})$ and the ratio of CR, PR, and stable disease, respectively. Stable duration was defined as the time from the first achievement of the best response to the time of PD. Adverse effects were evaluated using the Common Terminology Criteria for Adverse Events version 4.0 [19]. PFS was defined as the period from the day of Bev administration to the day of death or disease recurrence/progression. OS was defined as the period from the day of Bev administration to the day of death or the last confirmation of survival.

Statistical analysis was performed using JMP Pro 14 software (SAS Institute Inc., Cary, NC, USA). The chisquare test, Fisher's exact test, and Mann-Whitney U test were used to evaluate the clinical significance of clinicopathological factors. The frequency of adverse effects was compared between patients with grades 0,1 , and 2 and those with grades 3 and 4. PFS and OS curves were generated using the Kaplan-Meier method. Comparison 
Table 1 Details of drugs combined with bevacizumab

\begin{tabular}{lll}
\hline Combination drugs & Number of patients & Regimens \\
\hline Group A & $n=44$ & \\
Gemcitabine and oxaliplatin & 24 & $400 \mathrm{mg} / \mathrm{m}^{2}$ gemcitabine and $40 \mathrm{mg} / \mathrm{m}^{2}$ oxaliplatin on days 1,8 , and 15 \\
Pegylated liposomal doxorubicin & 10 & $10 \mathrm{mg} / \mathrm{m}^{2}$ pegylated liposomal doxorubicin on days 1,8 , and 15 \\
Eribulin and oxaliplatin & 5 & $1 \mathrm{mg} / \mathrm{m}^{2}$ eribulin and $40 \mathrm{mg} / \mathrm{m}^{2}$ oxaliplatin on days 1,8 , and 15 \\
Nivolumab & 1 & $100 \mathrm{mg} \mathrm{nivolumab}$ on days 1 and 15, every 4 weeks \\
Paclitaxel and carboplatin & 1 & $175 \mathrm{mg} / \mathrm{m}^{2}$ paclitaxel and AUC 5 carboplatin on day 1 \\
Paclitaxel & 1 & $80 \mathrm{mg} / \mathrm{m}^{2}$ paclitaxel on days 1,8 , and 15 \\
Trabectedin and oxaliplatin & 1 & $0.25 \mathrm{mg} / \mathrm{m}^{2}$ trabectedin and $40 \mathrm{mg} / \mathrm{m}^{2}$ oxaliplatin on days 1,8, and 15 \\
Trabectedin and pegylated liposomal doxoru- & 1 & $10 \mathrm{mg} / \mathrm{m}^{2}$ pegylated liposomal doxorubicin and $0.25 \mathrm{mg} / \mathrm{m}^{2}$ trabect- \\
bicin & $n=33$ & edin on days 1,8 , and 15 \\
Group B & 32 & $1000 \mathrm{mg} / \mathrm{m}^{2}$ gemcitabine on days 1,8 , and 15 \\
Gemcitabine & 1 & $80 \mathrm{mg} / \mathrm{m}^{2}$ paclitaxel on days 1,8, and 15 \\
Paclitaxel & 15
\end{tabular}

Group A was defined as patients treated with weekly low-dose administration of bevacizumab

Group B was defined as patients treated with monthly high-dose administration of bevacizumab

AUC Area under the curve

of PFS, OS, and stable duration was performed using the log-rank test. Cox proportional hazards analysis was used for multivariate analysis of PFS. Statistical significance was defined as a $p$-value of $<0.05$.

\section{Results}

Of the patients, 44 were in group A and 33 were in group B. More patients in group A had an advanced FIGO stage of the disease $(p=0.03)$, and a lower dose of Bev at the first time during the first cycle administration $(p<0.01)$ than in group B (Table 2). The best response, response rate, and clinical benefit rate were not statistically different, but the stable duration was longer in group A than in group B (8 vs. 5 months, $p<0.01$ ). The number of patients censored for OS was 26 in group A and 2 in group B $(p<0.01)$. The PFS of group A was better than that of group B (Fig. 1 A, $p<0.01$ ); however, the same was not true for OS (Fig. 1B, $p=0.58$ ). The number of patients censored for OS owing to treatment, follow-up, and dropout was 26/44 (59\%) in group A and 2/33 (6\%) in group B $(p<0.01)$. In the multivariate analysis for PFS, weekly low-dose administration of Bev was identified as an independent prognostic factor (Table 3; hazard ratio $0.53, p=0.03$ ).

There were fewer patients with grade $3 / 4$ anemia $(p<0.01)$, grade $3 / 4$ neutropenia $(p<0.01)$, grade $3 / 4$ hypertension $(p<0.01)$, intestinal obstruction $(p<0.01)$, and thromboembolic events $(p<0.01)$ in group A than in group B (Table 4). In group A, there were no statistical differences in PFS $(6,12$, and 5 months, respectively; $p<0.12)$, response rate $(20 \%, 30 \%$, and $10 \%$, respectively; $p=0.55)$, clinical benefit rate $(75 \%, 100 \%$, and $80 \%$, respectively; $p=0.32)$, and stable duration $(4,9.5$, and 3 months, respectively; $p<0.13$ ) among patients treated with gemcitabine and oxaliplatin, pegylated liposomal doxorubicin, and other regimens.

\section{Discussion}

The administration method of Bev was different among four clinical trials (GOG-218, ICON-7, OCEANS, and AURELIA) [4/7]. The method of Bev administration was $15 \mathrm{mg} / \mathrm{kg}$ body weight triweekly in GOG-218, $7.5 \mathrm{mg} / \mathrm{kg}$ triweekly in ICON-7, $15 \mathrm{mg} / \mathrm{kg}$ triweekly in OCEANS, and $10 \mathrm{mg} / \mathrm{kg}$ biweekly or $15 \mathrm{mg} / \mathrm{kg}$ triweekly in AURELIA. The duration of administration was similar across all clinical trials, except AURELIA. The AURELIA trial was the only trial that included patients who received different doses of Bev at different durations; however, the effects of these differences were unclear. Interestingly, although the dose of Bev in ICON-7 was half that in GOG-218, the ICON-7 trial demonstrated a long PFS, similar to GOG-218. Although we could not directly compare ICON-7 and GOG-218, we considered that there was still room for improvement in setting the dose of Bev. In fact, in our study, the group that received weekly low-dose Bev administration had a longer PFS. A low dose of Bev may be effective in patients with ovarian carcinoma.

The efficacy of angiogenesis inhibitors depends on the administration methods and dose in the preclinical model. Continuous treatment can normalize the tumor vasculature and transiently increase the drug uptake of the tumor. Conversely, intermittent treatment might allow the recovery of tumor vascularity between each 
Table 2 Characteristics of patients with ovarian carcinoma according to bevacizumab administration

\begin{tabular}{|c|c|c|c|c|c|}
\hline \multirow[t]{2}{*}{ Characteristics } & \multicolumn{2}{|l|}{ Group A } & \multicolumn{2}{|c|}{ Group B } & \multirow[b]{2}{*}{$p$-Value } \\
\hline & $n=44$ & & $n=33$ & & \\
\hline Age (years) & & & & & 0.07 \\
\hline Median (range) & 54 & $(30-77)$ & 61 & $(29-77)$ & \\
\hline FIGO stage (\%) & & & & & 0.03 \\
\hline I & 2 & $(4.5)$ & 7 & $(21.2)$ & \\
\hline$\|$ & 4 & $(9.1)$ & 3 & $(9.1)$ & \\
\hline III & 33 & $(75.0)$ & 15 & $(45.5)$ & \\
\hline IV & 5 & $(11.4)$ & 8 & $(24.2)$ & \\
\hline Histological types (\%) & & & & & 0.24 \\
\hline Serous carcinoma & 26 & $(59.1)$ & 18 & $(54.6)$ & \\
\hline Clear cell carcinoma & 5 & $(11.4)$ & 9 & $(27.3)$ & \\
\hline Endometrioid carcinoma & 6 & $(13.6)$ & 1 & $(3.0)$ & \\
\hline Carcinosarcoma & 1 & (2.3) & 1 & (3.0) & \\
\hline Squamous cell carcinoma & 1 & $(2.3)$ & 0 & $(0)$ & \\
\hline Mucinous carcinoma & 0 & (0) & 1 & $(3.0)$ & \\
\hline Adenocarcinoma not otherwise specified & 5 & $(11.4)$ & 3 & $(9.1)$ & \\
\hline Residual tumor ( $\geq 1 \mathrm{~cm}$ ) at primary surgery (\%) & & & & & 0.08 \\
\hline Yes & 22 & $(50.0)$ & 10 & $(30.3)$ & \\
\hline No & 22 & $(50.0)$ & 23 & $(69.7)$ & \\
\hline $\begin{array}{l}\text { Prior chemotherapy regimens before bevacizumab-con- } \\
\text { taining regimens (times) }\end{array}$ & 3.8 & $(1-11)$ & 3.5 & $(1-11)$ & 0.61 \\
\hline $\begin{array}{l}\text { Total dose of bevacizumab set for the first time during the } \\
\text { first cycle (mg) }\end{array}$ & 400.0 & $(400)$ & 745.6 & $(520-1113)$ & $<0.01$ \\
\hline Best response & & & & & 0.13 \\
\hline Complete remission & 3 & $(6.8)$ & 0 & $(0)$ & \\
\hline Partial remission & 6 & $(13.6)$ & 6 & $(18.2)$ & \\
\hline Stable disease & 27 & $(61.4)$ & 15 & $(45.5)$ & \\
\hline Progressive disease & 8 & $(18.2)$ & 12 & $(36.4)$ & \\
\hline Response rate (\%) & 20 & & 18.2 & & 0.52 \\
\hline Clinical benefit rate (\%) & 82 & & 63.6 & & 0.07 \\
\hline Stable duration (months) & 8 & $(0-37)$ & 5 & $(0-10)$ & $<0.01$ \\
\hline
\end{tabular}

Group A was defined as patients treated with weekly low-dose administration of bevacizumab Group B was defined as patients treated with monthly high-dose administration of bevacizumab FIGO International Federation of Gynecology and Obstetrics

cycle of drug administration and might facilitate tumor cell recovery from cytotoxic drug treatment [10]. Moreover, lower doses of angiogenesis inhibitors might improve perfusion and drug delivery better than higher doses [11]. In fact, a randomized phase II trial of treatment for metastatic breast carcinoma demonstrated no survival benefit, and differences in response rates were observed among patients who received Bev every week $(10 \mathrm{mg} / \mathrm{kg})$, every 2 weeks $(10 \mathrm{mg} / \mathrm{kg})$, and every 3 weeks $(15 \mathrm{mg} / \mathrm{kg})$ as the co-administered drug of nanoparticle albumin-bound paclitaxel [20]. Meanwhile, a randomized phase II trial of treatment for metastatic colon carcinoma demonstrated that patients who received a lower dose of Bev $(5 \mathrm{mg} /$ kg every 2 weeks) had a survival benefit over those who received a higher dose of Bev (10 mg/kg every 2 weeks) as the co-administered drug of fluorouracil and leucovorin [21]. Our study showed that weekly administration of a lower dose of Bev might be effective for platinum-resistant recurrent ovarian carcinoma, as observed for colon carcinoma. The efficacy of the administration methods of Bev might differ among several carcinomas.

In our study, most patients in the monthly high-dose administration group received gemcitabine as the coadministered drug with Bev, whereas patients in the weekly low-dose Bev group received various co-administered agents, which could have affected our results. In a phase III trial examining the efficacy of gemcitabine monotherapy in patients with platinum-resistant 

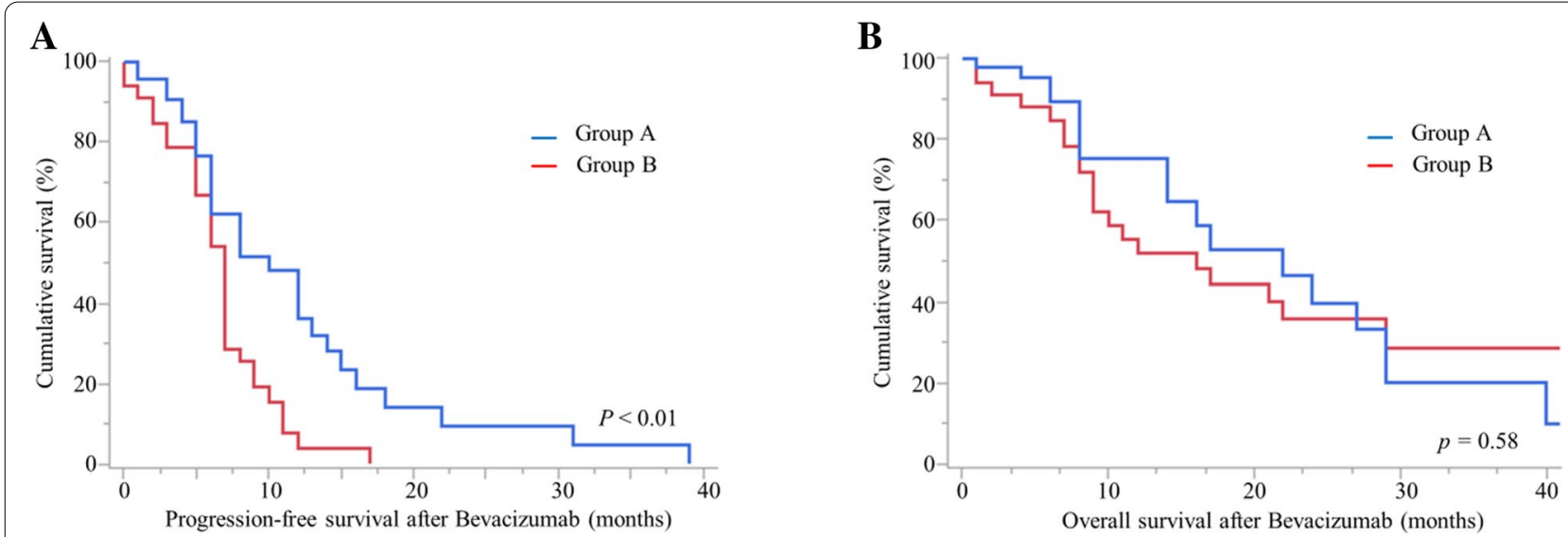

Fig. 1 Progression-free survival (A) and overall survival (B). Group A was defined as patients treated with weekly low-dose administration of bevacizumab. Group $\mathbf{B}$ was defined as patients treated with monthly high-dose administration of bevacizumab

Table 3 Univariate and multivariate analyses for progression-free survival after bevacizumab administration in all cases included in this study

\begin{tabular}{|c|c|c|c|c|c|}
\hline \multirow[b]{2}{*}{ Variables } & & \multicolumn{2}{|l|}{ Univariate analysis } & \multicolumn{2}{|l|}{ Multivariate analysis } \\
\hline & & Hazard ratio $(95 \% \mathrm{Cl})$ & $p$-Value & Hazard ratio $(95 \% \mathrm{CI})$ & $p$-Value \\
\hline Method of bevacizumab administration & Group A vs. group B & $0.47(0.27-0.80)$ & $<0.01$ & $0.53(0.29-0.95)$ & 0.03 \\
\hline Age (years) & $\geq 50$ vs. $<50$ & $1.83(1.01-3.50)$ & 0.045 & $1.40(0.73-2.81)$ & 0.31 \\
\hline Drug combined with bevacizumab & Gemcitabine vs. other regimens & $1.70(0.95-3.25)$ & 0.08 & & \\
\hline Histology & Serous carcinoma vs. other histology & $0.73(0.43-1.24)$ & 0.24 & & \\
\hline Residual tumor at primary surgery & $<1 \mathrm{~cm}$ vs. $\geq 1 \mathrm{~cm}$ & $0.94(0.55-1.58)$ & 0.82 & & \\
\hline FIGO stage & I, II vs. III, IV & $0.98(0.54-1.90)$ & 0.95 & & \\
\hline
\end{tabular}

Group A was defined as patients treated with weekly low-dose administration of bevacizumab

Group B was defined as patients treated with monthly high-dose administration of bevacizumab

$\mathrm{Cl}$ confidence interval, FIGO International Federation of Gynecology and Obstetrics

recurrence, the overall response rate was $6.1 \%$ and the median PFS was 3.6 months [22]. In a multicenter phase II clinical trial investigating the combination therapy of gemcitabine and oxaliplatin in patients with platinumresistant recurrent ovarian cancer, the overall response rate was $37 \%$ and the median PFS was 6.7 months [23]. In a phase III trial determining the effect of pegylated liposomal doxorubicin in patients with platinum-refractory and platinum-resistant ovarian cancer, the overall response rate was $17.4 \%$ and the median PFS was 4.0 months [24]. Our study confirmed that there were no statistical differences in efficacy in the group treated with a weekly low-dose administration of Bev. Furthermore, survival analysis demonstrated that the co-administered drugs were not prognostic factors. Thus, the results of our study might be greatly affected by the weekly low doses of Bev, but not by the co-administered drugs.

Several clinical trials have reported that hypertension, thromboembolic events, and gastrointestinal perforation are well-known adverse events associated with Bev
[4-7]. In our study, the incidences of these adverse events were lower in the group treated with weekly low-dose administration of Bev, in addition to a lower incidence of hematological toxicities. Therefore, weekly low-dose administration of Bev might decrease the frequency of adverse effects while increasing the anti-tumor effect.

Anti-angiogenic drugs had a synergic effect with poly (ADP-ribose) polymerase inhibitors in the PAOLA-1 trial [9]. In addition, the combination of immune-checkpoint inhibitors and Bev was considered to be promising because VEGF-mediated immunosuppressive effects enhanced immune-mediated antitumor activity [25]. In fact, phase Ib and II trials showed promising effects and tolerable adverse effects. Therefore, anti-angiogenic drugs such as Bev play a central role in chemotherapy for ovarian carcinoma [26, 27]. Future studies should investigate the method of administration of anti-angiogenic drugs.

Our study had several limitations, including its retrospective nature and small sample size. In particular, we 
Table 4 Comparison of adverse effects between the weekly bevacizumab group and the monthly bevacizumab group

\begin{tabular}{|c|c|c|c|c|c|}
\hline \multirow[t]{2}{*}{ Adverse effects } & \multicolumn{2}{|c|}{ Group A $(n=44)$} & \multicolumn{2}{|c|}{ Group B $(n=33)$} & \multirow[t]{2}{*}{$p$-Value } \\
\hline & Grade $1 / 2$ & Grade $3 / 4$ & Grade $1 / 2$ & Grade $3 / 4$ & \\
\hline Anemia & 41 & 2 & 20 & 12 & $<0.01$ \\
\hline Neutropenia & 22 & 10 & 12 & 18 & $<0.01$ \\
\hline Thrombocytopenia & 29 & 2 & 14 & 1 & 0.99 \\
\hline Febrile neutropenia & 0 & 0 & 0 & 0 & 0.99 \\
\hline aspartate aminotransferase level increased & 0 & 1 & 4 & 1 & 0.99 \\
\hline alanine aminotransferase level increased & 2 & 0 & 2 & 0 & 0.99 \\
\hline Fatigue & 37 & 0 & 11 & 0 & 0.99 \\
\hline Stomatitis & 11 & 0 & 0 & 0 & 0.99 \\
\hline Nausea & 32 & 1 & 10 & 2 & 0.57 \\
\hline Hypertension & 5 & 1 & 4 & 11 & $<0.01$ \\
\hline Thromboembolic event & 1 & 0 & 2 & 6 & $<0.01$ \\
\hline Proteinuria & 3 & 0 & 9 & 1 & 0.43 \\
\hline Gastrointestinal perforation & 0 & 0 & 0 & 2 & 0.57 \\
\hline Intestinal obstruction & 0 & 0 & 4 & 6 & $<0.01$ \\
\hline Cardiac disorder & 0 & 0 & 1 & 0 & 0.99 \\
\hline Hand and foot syndrome & 10 & 0 & 0 & 0 & 0.99 \\
\hline Constipation & 15 & 0 & 2 & 0 & 0.99 \\
\hline Diarrhea & 3 & 0 & 1 & 1 & 0.43 \\
\hline Taste disorder & 3 & 0 & 1 & 0 & 0.99 \\
\hline
\end{tabular}

Group A was defined as patients treated with weekly low-dose administration of bevacizumab

Group B was defined as patients treated with monthly high-dose administration of bevacizumab

could not perform a comfortable analysis of OS. However, our study did not have selection bias in terms of the two administration methods of Bev. Furthermore, weekly low-dose administration of Bev was found to improve PFS and decrease the adverse effects associated with this drug. Thus, we believe that this information needs to be immediately shared and a future study examining the administration methods of Bev is warranted.

\section{Conclusions}

In conclusion, weekly low-dose administration of Bev might be the potential treatment for patients with platinum-resistant ovarian carcinoma in terms of survival benefit and adverse effects. It might be worth performing further studies to examine the methods of administration of anti-angiogenic drugs.

\section{Abbreviations}

AURELIA: A Study of Bevacizumab Added to Chemotherapy in Patients With Platinum-resistant Ovarian Cancer; BRCA: Breast cancer susceptibility gene; Bev: Bevacizumab; FIGO: International Federation of Gynecology and Obstetrics; GOG: Gynecologic Oncology Group; PFS: Progression-free survival; ICON: International Collaboration on Ovarian Neoplasms; OCEANS: The Ovarian Cancer Study Comparing Efficacy and Safety of Chemotherapy and Anti-angiogenic Therapy in Platinum-Sensitive Recurrent Disease; OS: Overall survival; PAOLA: Randomized, Double-Blind Controll Trial of Olaparib vs. Placebo in Patients with Advanced Ovarian Cancer; VEGF: Humanized vascular endothelial factor.

\section{Acknowledgements}

We would like to thank Editage (www.editage.jp) for English language editing.

\section{Authors' contributions}

Protocol/project development: JS, MM, YK, and MT. Data collection or management: TY, HK, TH, HI, TI, HI, and SK. Data analysis: JS, RS, TS, HM, and NK. Manuscript writing/editing: JS, MM, YK, and MT. All authors have read and approved the manuscript.

\section{Funding}

This study was conducted without any financial support.

Availability of data and materials

The corresponding author send dataset according to reasonable request.

\section{Declarations}

\section{Ethics approval and consent to participate}

This study was approved by the institutional review board of the National Defense Medical College and Ohki Memorial Kikuchi Cancer Clinic for Women, Tokorozawa, Japan. The records and information of all women included in the study were anonymized and de-identified before the analysis. Informed consent did not be obtained from all participants due to retrospective analysis.

\section{Consent for publication}

Not applicable.

\section{Competing interests}

The authors declare that they have no conflicts of interest.

\section{Author details}

${ }^{1}$ Department of Obstetrics and Gynecology, National Defense Medical College Hospital, 3-2, Namiki, Tokorozawa, Saitama 359-8513, Japan. ²Department of Clinical Oncology, National Defense Medical College Hospital, 3-2, Namiki, 
Tokorozawa, Saitama 359-8513, Japan. ${ }^{3}$ Department of Gynecology, Ohk Memorial Kikuchi Cancer Clinic for Women, 111-1, Arahata, Tokorozawa, Saitama 359-1133, Japan.

Received: 27 September 2021 Accepted: 7 February 2022 Published online: 16 February 2022

\section{References}

1. Siegel RL, Miller KD, Jemal A. Cancer statistics, 2019. CA Cancer J Clin. 2019;69:7-34

2. Lheureux S, Gourley C, Vergote I, Oza AM. Epithelial ovarian cancer. Lancet. 2019;393:1240-53.

3. Presta LG, Chen H, O'Connor SJ, Chisholm V, Meng YG, Krummen L, Winkler M, et al. Humanization of an anti-vascular endothelial growth factor monoclonal antibody for the therapy of solid tumors and other disorders. Cancer Res. 1997:57:4593-9.

4. Burger RA, Brady MF, Bookman MA, Fleming GF, Monk BJ, Huang H, et al. Incorporation of bevacizumab in the primary treatment of ovarian cancer. N Engl J Med. 2011;365:2473-83.

5. Perren TJ, Swart AM, Pfisterer J, Ledermann JA, Pujade-Lauraine E, Kristensen $\mathrm{G}$, et al. A phase 3 trial of bevacizumab in ovarian cancer. N Engl J Med. 2011:365:2484-96.

6. Aghajanian C, Blank SV, Goff BA, Judson PL, Teneriello MG, Husain A, et al. OCEANS: a randomized, double-blind, placebo-controlled phase III trial of chemotherapy with or without bevacizumab in patients with platinumsensitive recurrent epithelial ovarian, primary peritoneal, or fallopian tube cancer. J Clin Oncol. 2012;30:2039-45.

7. Pujade-Lauraine E, Hilpert F, Weber B, Reuss A, Poveda A, Kristensen G, et al. Bevacizumab combined with chemotherapy for platinum-resistant recurrent ovarian cancer: the AURELIA open-label randomized phase III trial. J Clin Oncol. 2014:32:1302-8.

8. Poveda AM, Selle F, Hilpert F, Reuss A, Savarese A, Vergote I, et al. Bevacizumab combined with weekly paclitaxel, pegylated liposomal doxorubicin, or topotecan in platinum-resistant recurrent ovarian cancer: analysis by chemotherapy cohort of the randomized phase III AURELIA trial. J Clin Oncol. 2015;33:3836-8.

9. Ray-Coquard I, Pautier P, Pignata S, Pérol D, González-Martín A, Berger $R$, et al. Olaparib plus bevacizumab as first-line maintenance in ovarian cancer. N Engl J Med. 2019;381:2416-28.

10. Ma J, Waxman DJ. Combination of antiangiogenesis with chemotherapy for more effective cancer treatment. Mol Cancer Ther. 2008;7:3670-84.

11. Huang Y, Yuan J, Righi E, Kamoun WS, Ancukiewicz M, Nezivar J, et al. Vascular normalizing doses of antiangiogenic treatment reprogram the immunosuppressive tumor microenvironment and enhance immunotherapy. Proc Natl Acad Sci U S A. 2012;109:17561-6.

12. Kerbel RS, Kamen BA. The anti-angiogenic basis of metronomic chemotherapy. Nat Rev Cancer. 2004;4:423-36.

13. Saito K, Kikuchi Y, Fujii K, Kita T, Furuya K. Effect of paclitaxel on vascular endothelial growth factor (VEGF) and interleukin (IL)-8 in serum of patients with recurrent ovarian cancer: a comparison of weekly vs triweekly regimens. Target Oncol. 2006;1:86-9.

14. Pignata S, Scambia G, Katsaros D, Gallo C, Pujade-Lauraine E, De Placido $\mathrm{S}$, et al. Carboplatin plus paclitaxel once a week versus every 3 weeks in patients with advanced ovarian cancer (MITO-7): a randomised, multicentre, open-label, phase 3 trial. Lancet Oncol. 2014;15:396-405.

15. Katsumata N, Yasuda M, Takahashi F, Isonishi S, Jobo T, Aoki D, et al. Dosedense paclitaxel once a week in combination with carboplatin every 3 weeks for advanced ovarian cancer: a phase 3, open-label, randomised controlled trial. Lancet. 2009;374:1331-8.

16. Takasaki K, Miyamoto M, Takano M, Soyama H, Aoyama T, Matsuura H, et al. Addition of bevacizumab to gemcitabine for platinum-resistant recurrent ovarian cancer: a retrospective analysis. Cancer Chemother Pharmacol. 2008:81:809-14

17. Pereira A, Pérez-Medina T, Magrina JF, Magtibay PM, Rodríguez-Tapia A, Peregrin I, et al. International Federation of Gynecology and Obstetrics staging classification for cancer of the ovary, fallopian tube, and peritoneum: estimation of survival in patients with node-positive epithelial ovarian cancer. Int J Gynecol Cancer. 2015;25:49-54.
18. Eisenhauer EA, Therasse P, Bogaerts J, Schwartz LH, Sargent D, Ford R, et al. New response evaluation criteria in solid tumours: revised RECIST guideline (version 1.1). Eur J Cancer. 2009;45(2):228-47.

19. National Cancer Institute, National Institutes of Health, US Department of Health and Human Services. Common Terminology Criteria for Adverse Events (CTCAE), version 4.0. National Cancer Institute. 2021. https://ctep. cancer.gov/protocoldevelopment/electronic_applications/ctc.htm\#ctc_ 40. Accessed 20 Jan 2021.

20. Seidman AD, Conlin AK, Bach A, Moynahan ME, Lake D, Forero A, et al. Randomized phase II trial of weekly vs. every 2 weeks vs. every 3 weeks nanoparticle albumin-bound paclitaxel with bevacizumab as firstline chemotherapy for metastatic breast cancer. Clin Breast Cancer. 2013;13(4):239-46.e1.

21. Kabbinavar F, Hurwitz HI, Fehrenbacher L, Meropol NJ, Novotny WF, Lieberman $\mathrm{G}$, et al. Phase II, randomized trial comparing bevacizumab plus fluorouracil (FU)/leucovorin (LV) with FU/LV alone in patients with metastatic colorectal cancer. J Clin Oncol. 2003;21:60-5.

22. Mutch DG, Orlando M, Goss T, Teneriello MG, Gordon AN, McMeekin SD, et al. Randomized phase III trial of gemcitabine compared with pegylated liposomal doxorubicin in patients with platinum-resistant ovarian cancer. J Clin Oncol. 2007;25:2811-8.

23. Vici P, Sergi D, Pizzuti L, Mariani L, Arena MG, Barba M, et al. Gemcitabineoxaliplatin (GEMOX) as salvage treatment in pretreated epithelial ovarian cancer patients. J Exp Clin Cancer Res. 2013;32:49.

24. Motohashi T, Yabuno A, Michimae H, Ohishi T, Nonaka M, Takano M, et al. Randomized phase III trial comparing pegylated liposomal doxorubicin (PLD) at $50 \mathrm{mg} / \mathrm{m}^{2}$ versus $40 \mathrm{mg} / \mathrm{m}^{2}$ in patients with platinum-refractory and -resistant ovarian carcinoma: the JGOG 3018 Trial. J Gynecol Oncol. 2021;32(1):e9.

25. Yi M, Jiao D, Qin S, Chu Q, Wu K, Li A. Synergistic effect of immune checkpoint blockade and anti-angiogenesis in cancer treatment. Mol Cancer. 2019;18:60

26. Liu JF, Herold C, Gray KP, Penson RT, Horowitz N, Konstantinopoulos PA, et al. Assessment of combined nivolumab and bevacizumab in relapsed ovarian cancer: a phase 2 clinical trial. JAMA Oncol. 2019;5:1731-8.

27. Moroney JW, Powderly J, Lieu CH, Bendell JC, Eckhardt SG, Chang CW et al. Safety and clinical activity of atezolizumab plus bevacizumab in patients with ovarian cancer: a phase Ib study. Clin Cancer Res. 2020;26:5631-7.

\section{Publisher's Note}

Springer Nature remains neutral with regard to jurisdictional claims in published maps and institutional affiliations.

Ready to submit your research? Choose BMC and benefit from:

- fast, convenient online submission

- thorough peer review by experienced researchers in your field

- rapid publication on acceptance

- support for research data, including large and complex data types

- gold Open Access which fosters wider collaboration and increased citations

- maximum visibility for your research: over 100M website views per year

At BMC, research is always in progress.

Learn more biomedcentral.com/submissions 